\section{A rationale for membrane peeling in the repair of stage 4 macular holes}

${ }^{1}$ Vitreoretinal Service, Addenbrooke's NHS Trust, Hills Road, Cambridge UK

\section{${ }^{2}$ Department of Histopathology, University Hospitals of Coventry and Warwickshire NHS Trust, Coventry UK}

Correspondence: MP Snead Vitreoretinal Service, BOX 41, Addenbrooke's NHS Trust, Hills Road, Cambridge CB2 2QQ, UK

Tel: + 441223 216701;

Fax: +44 1223217968.

E-mail: mps34@

cam.ac.uk

Received: 10 October 2004 Accepted in revised form: 31 January 2005 Published online: 1 April 2005

\section{Abstract}

Aim To examine the histological and immunocytochemical characteristics of epiretinal membranes (ERM) associated with stage 4 macular holes (MH) so as to establish a vitreoretinal rationale for surgery in stage 4 MH.

Method Consecutive patients with stage 4 MH undergoing vitrectomy and membrane peeling were recruited. Preoperatively, the eyes were examined for ERM formation over the macula and completeness of posterior hyaloid membrane (PHM) separation from the retina. ERM peel specimens obtained during surgery were sent for histological and immunocytochemical studies and were compared with the PHM specimens taken from a previous post-mortem study of eyes with physiological posterior vitreous detachment but without macular holes.

Result A total of 13 patients with stage $4 \mathrm{MH}$ fulfilled the inclusion criteria and were recruited. Preoperatively, all eyes had an ERM over the macula and incomplete separation of the PHM seen as a defect in the PHM on specular biomicroscopy. Histologically, the ERM specimens had very similar morphological characteristics to PHM, consisting of an eosinophilic membrane of varying thickness with scattered spindleshaped cells. The membranes stained positively for type IV collagen while the cells were glial fibrillary acidic protein positive.

Postoperatively, successful closure of MH was achieved in all cases.

Conclusion Stage $4 \mathrm{MH}$ is characterised by incomplete separation of the PHM from the retina with remnants overlying the macula manifesting as ERM. Removal of the ERM is required during vitrectomy in order to relieve the tangential forces involved in the development of MH.
A Ang', DRJ Snead², S James², YC Yeo², K Satchi', AHC Morris' ${ }^{1}$, AV Poulson' ${ }^{1}$, JD Scott ${ }^{1}$ and MP Snead'

Eye (2006) 20, 208-214. doi:10.1038/sj.eye.6701856; published online 1 April 2005

Keywords: macular hole; outcome; vitrectomy; posterior vitreous detachment; epiretinal membrane; peel

\section{Introduction}

Idiopathic macular hole (MH) is a rare condition that affects mainly women in their seventh decade. It has been proposed that MH develops as a result of prefoveal cortical vitreous contraction, causing tangential forces that dehisces the neurosensory retina at the fovea. ${ }^{1}$

The role of surgery in macular hole repair has traditionally been considered to eliminate tangential surface traction at the vireoretinal interface and there is clinical, OCT, and surgical evidence to support this hypothesis. ${ }^{1-6}$ In stage 4 holes, however, the rationale for vitrectomy surgery becomes less easy to extrapolate since the vitreous has (by definition) already separated from the retina. It also calls into question why such holes do not more commonly undergo spontaneous closure.

Genuine posterior vitreous detachment (PVD) should be defined as the separation of not only the cortical gel but in addition, the posterior hyaloid membrane (PHM). ${ }^{7}$ This study was designed to test our hypothesis that stage 4 holes are characterised by a 'rhexis' in, and incomplete separation of, the PHM from the retinal surface with PHM remnants still attached to the macula. Our study compares the histological and immunocytochemical characteristics of the residual surface membrane removed from stage $4 \mathrm{MHs}$ with those of the PHM from physiological, uncomplicated PVD in order to provide a rationale for vitrectomy and membrane peeling in stage $4 \mathrm{MHs}$. 


\section{Method}

Patients referred to the Vitreoretinal Service at Addenbrooke's Hospital for MH surgery were invited to participate in the study following informed consent. Only patients with stage $4 \mathrm{MH}$ were included in this project. No time restriction was imposed for the prior duration of symptoms and all patients who chose to undergo surgery were included so that cases were consecutive and unselected. The study was approved by the local ethics committee.

Preoperative data collected for each patient included age and sex of patient, past ophthalmic history, visual symptoms encountered, and the duration of those symptoms. Each patient underwent a complete ophthalmological examination including best-corrected Snellen acuity, slit-lamp examination, and biomicroscopic examination of the vitreous, PHM, and its relationship to the fovea/retina.

An MH was diagnosed when a full-thickness retinal dehiscence involving primarily the foveola was seen on biomicroscopy. The presence of a PVD was confirmed by the identification of the PHM, characterised by a mobile and refractile membrane bordering the posterior vitreous gel. It was best identified by specular, dynamic biomicroscopy with the slit-lamp light source offset by about $10^{\circ}$. The presence or absence of a defect in the PHM was also documented. The defect was seen as a scimitar 'capsulorhexis-like' defect, with cortical gel spilling through posteriorly into the preretinal space (Figure 1).

Vitrectomy was carried out with a three-port technique. The epiretinal membrane (ERM) (resulting from residual attached PHM over the macula) was removed using a $25 \mathrm{G}$ needle or a pair of forceps. The membrane was sent for histology and

immunocytochemistry. Prior to closure, a fluid-gas (14\% perfluoropropane $\left(\mathrm{C}_{3} \mathrm{~F}_{8}\right)$ ) exchange was carried out and

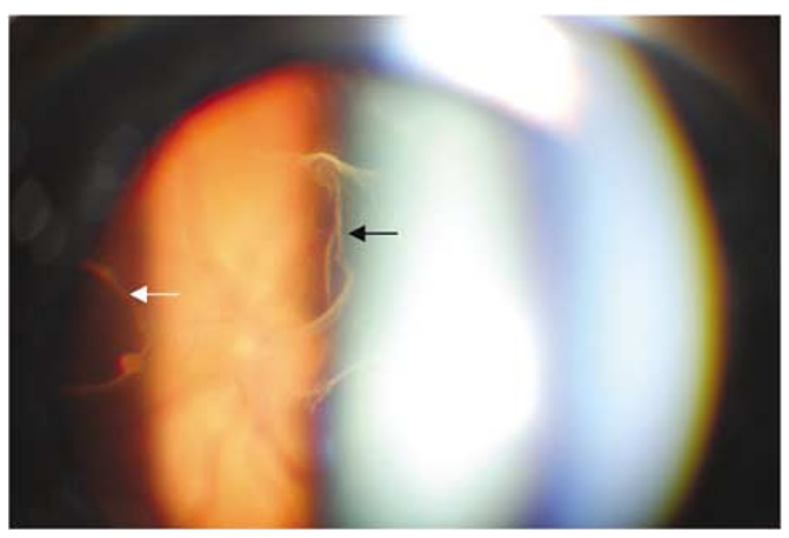

Figure 1 Posterior hyaloid membrane (PHM) with Weiss ring (white arrow) and PHM defect (black arrow). the patients were advised to posture face down for 2 weeks postoperatively. In the postoperative period, information on the anatomical outcome, best-corrected visual acuity and the development of any postoperative complications were obtained from the latest outpatient visit.

To act as controls, patients with physiological PVD and without $\mathrm{MH}$ were studied from an unselected group of general medical in-patients as previously reported. ${ }^{7}$ In brief, patients were recruited from general medical wards with informed written consent and were given a full ophthalmological examination to identify the presence of a PVD as described above. In the event of the patient's death, his/her eyes were enucleated and fixed in $10 \%$ buffered formal saline before preparation for histological and immunocytochemical examination of the PHM.

\section{Specimen processing}

The specimens from $\mathrm{MH}$ surgery were fixed in formalin. The visible membranes were removed from the specimen pots and placed directly into liquid agar (agar gel dissolved in $10 \%$ formalin and heated with a microwave oven). After setting, these agar blocks were placed into tissue cassettes and processed into paraffin wax blocks using a standard tissue processor (PathCenter, Shandon Scientific Ltd, Chadwick Rd., Runcorn, Cheshire, UK). Consecutive ribbons of $5 \mu \mathrm{m}$ sections were cut, placed on seven coated slides, and stained with haematoxylin and eosin (H\&E) or immunocytochemical stains (see below).

The control specimens were processed in one of two ways. Firstly, five globes were processed into oversized paraffin blocks as previously described. ${ }^{7}$ These globes were sectioned in para-sagittal planes giving central, temporal, and nasal callotes. Sections were cut at a number of levels including through the optic disc and stained with H\&E and alcian blue (to highlight the vitreous gel). In the second group (two globes), a $13 \mathrm{~mm}$ trephine centred on the optic nerve was used to remove a disc of posterior sclera, choroids, and retina, giving access to the posterior aspect of the PHM. The PHM was retrieved intact and fixed in $10 \%$ buffered formal saline before being air-dried on coated slides. This was stained with H\&E.

Immunocytochemistry was carried out for type II and type IV collagen, low and high molecular weight cytokeratin (MNF 116) and glial fibrillary acidic protein (GFAP) using a standard immunoperoxidase technique according to the manufacturer's instructions. Sections for type II collagen staining were predigested with trypsin while antigen retrieval for the remaining antibodies was achieved by micro-waving the sections in buffer. In all cases, the avidin-biotin complex method with 
diaminobenzidine was used to visualise bound antibody. Meyers haematoxylin was used as counterstain. Negative control sections, omitting the primary antibody step, were run alongside test sections. Positive control sections of human tissues known to express the epitope being demonstrated, that is, GFAP-spinal cord, MNF116appendix, Collagen II-chondrosarcoma, and Collagen IV-pyogenic granuloma, were also run alongside the test sections.

\section{Results}

A total of 13 patients with stage $4 \mathrm{MH}$ of varying durations underwent surgery and were therefore recruited into the study. Patient demographics, surgical procedures, and outcomes are summarised in Table 1. The average age of the patients was 66 years. There were seven females and six males. The duration of preoperative symptoms averaged 16 months (range of 1-60 months). One patient (patient 11) was highly myopic with a spherical equivalent of -11 dioptres and one patient (patient 9) had previous surgery for retinal detachment not involving the fovea. Best corrected preoperative visual acuity ranged from $6 / 18$ to $3 / 60$.

On preoperative examination, all patients had evidence of PVD with the presence of a visible PHM. All patients also demonstrated a defect in the PHM with ERM surrounding the $\mathrm{MH}$.
Postoperatively, successful MH closure was achieved in all cases with improvement of best-corrected Snellen visual acuity at final follow-up (Table 1). The mean follow-up duration was 16 months (range: 6 months to 3 years). Three patients required subsequent cataract surgery (patients 7, 10, and 13) and one patient (patient 2) developed retinal detachment postoperatively.

\section{Histology and immunocytochemistry}

Six out of 13 membrane peel specimens were successfully collected during surgery and submitted for histological examination. Only three specimens survived processing for histology. All exhibited a diaphanous, eosinophilic membrane of varying thickness, ranging from 2 to $25 \mu \mathrm{m}$, and studded with scattered spindle shaped cells

(Figure 2). The cells usually appeared to be lining one side of the membrane, although some examples appeared to show cells embedded in the membrane. They had round or oval nuclei and were sparse in number, appearing quite widely spaced along the membrane. No other cell types (including retinal pigment epithelial cells, photoreceptors and inflammatory cells) were identified by routine stains.

Immunocytochemistry showed the membrane specimens to be positive for type IV collagen (Figure 3a). Type II collagen stain was weakly positive and appeared

Table 1 Summary of demographics, surgical procedures, and outcomes of recruited patients

\begin{tabular}{|c|c|c|c|c|c|c|}
\hline No. & Age (years) & History & Operation & $\begin{array}{l}\text { Preoperative } \\
\text { vision }\end{array}$ & $\begin{array}{l}\text { Postoperative } \\
\text { vision }\end{array}$ & $\begin{array}{l}\text { Follow-up } \\
\text { months }\end{array}$ \\
\hline 1 & 84 & 1 month reduced reading vision. & $\mathrm{PPV}^{\mathrm{a}}$ and $\mathrm{C}_{3} \mathrm{~F}_{8}$ gas $^{\mathrm{b}}$ & $6 / 18$ & $6 / 9$ & 36 \\
\hline 2 & 69 & $\begin{array}{l}36 \text { months reduced reading vision. } \\
\text { Distortion }\end{array}$ & $\mathrm{PPV}$ and $\mathrm{C}_{3} \mathrm{~F}_{8}$ gas & $6 / 60$ & $6 / 24$ & 6 \\
\hline 3 & 56 & $\begin{array}{l}6 \text { months reduced reading vision. } \\
\text { Distortion }\end{array}$ & $\mathrm{PPV}$ and $\mathrm{C}_{3} \mathrm{~F}_{8}$ gas & $6 / 60$ & $6 / 18$ & 36 \\
\hline 4 & 60 & $\begin{array}{l}6 \text { months reduced reading vision. } \\
\text { Distortion }\end{array}$ & $\mathrm{PPV}$ and $\mathrm{C}_{3} \mathrm{~F}_{8}$ gas & $6 / 18$ & $6 / 9$ & 24 \\
\hline 5 & 69 & $\begin{array}{l}12 \text { months reduced reading vision. } \\
\text { Distortion }\end{array}$ & $\mathrm{PPV}$ and $\mathrm{C}_{3} \mathrm{~F}_{8}$ gas & $3 / 60$ & $6 / 18$ & 9 \\
\hline 6 & 64 & $\begin{array}{l}8 \text { months reduced reading vision. } \\
\text { Distortion }\end{array}$ & $\mathrm{PPV}$ and $\mathrm{C}_{3} \mathrm{~F}_{8}$ gas & $6 / 60$ & $6 / 36$ & 12 \\
\hline 7 & 73 & $\begin{array}{l}60 \text { months reduced reading vision. } \\
\text { Distortion }\end{array}$ & $\mathrm{PPV}$ and $\mathrm{C}_{3} \mathrm{~F}_{8}$ gas & $6 / 24$ & $6 / 6$ & 24 \\
\hline 8 & 67 & $\begin{array}{l}24 \text { months reduced reading vision. } \\
\text { Distortion }\end{array}$ & $\mathrm{PPV}$ and $\mathrm{C}_{3} \mathrm{~F}_{8}$ gas & $6 / 60$ & $6 / 24$ & 14 \\
\hline 9 & 54 & $\begin{array}{l}24 \text { months reduced reading vision. } \\
\text { Distortion }\end{array}$ & $\begin{array}{l}\text { Phaco }+\mathrm{IOL}^{\mathrm{c}}, \mathrm{PPV} \\
\text { and } \mathrm{C}_{3} \mathrm{~F}_{8} \text { gas }\end{array}$ & $6 / 60$ & $6 / 18$ & 18 \\
\hline 10 & 68 & 12 months reduced reading vision & $\mathrm{PPV}$ and $\mathrm{C}_{3} \mathrm{~F}_{8}$ gas & $6 / 60$ & $6 / 36$ & 12 \\
\hline 11 & 66 & 4 months reduced reading vision & $\mathrm{PPV}$ and $\mathrm{C}_{3} \mathrm{~F}_{8}$ gas & $3 / 60$ & $6 / 18$ & 6 \\
\hline 12 & 66 & 9 months reduced reading vision & $\mathrm{PPV}$ and $\mathrm{C}_{3} \mathrm{~F}_{8}$ gas & $6 / 18$ & $6 / 9$ & 6 \\
\hline 13 & 65 & 9 months reduced reading vision & $\mathrm{PPV}$ and $\mathrm{C}_{3} \mathrm{~F}_{8}$ gas & $3 / 60$ & $6 / 24$ & 6 \\
\hline
\end{tabular}

aPars plana vitrectomy.

bPerfluoropropane.

'Phacoemulsification and intraocular lens implantation. 

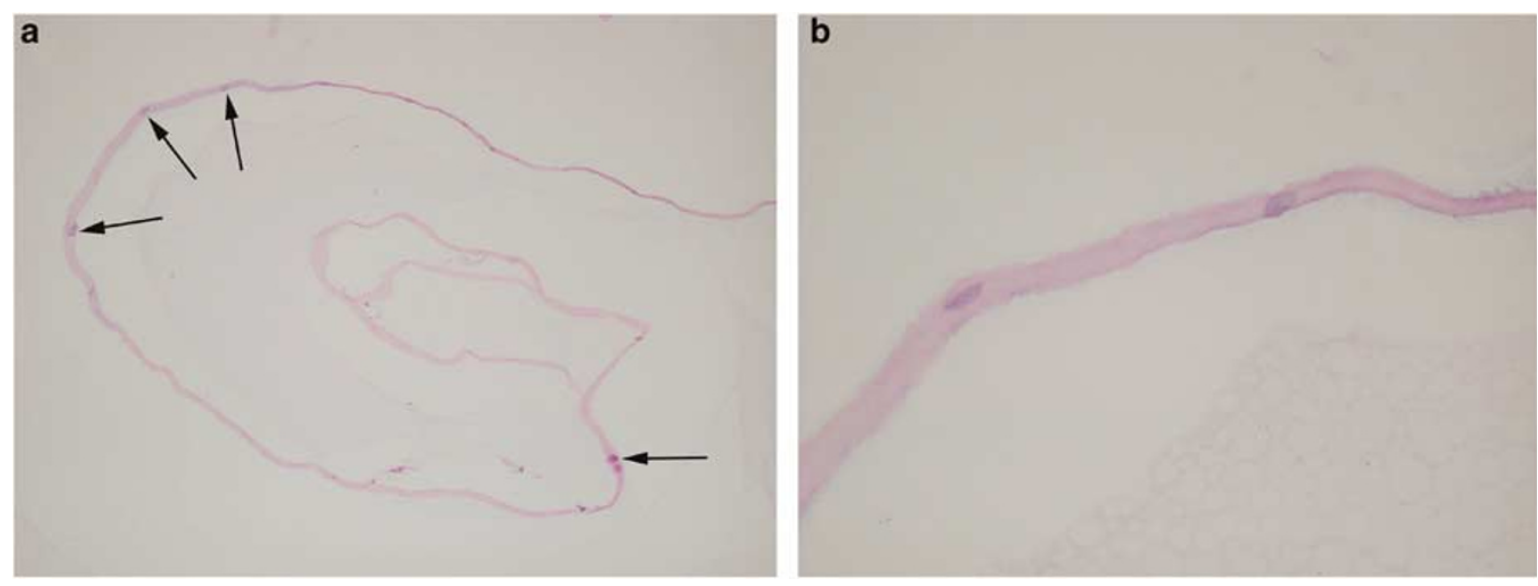

Figure 2 Histology of epiretinal membrane: (a) (H\&E) thin strip of eosinophilic membrane with scattered spindle-shaped cells (nuclei arrowed) (b) (H\&E) higher-power view. Magnifications (a) $\times 100$, (b) $\times 400$.
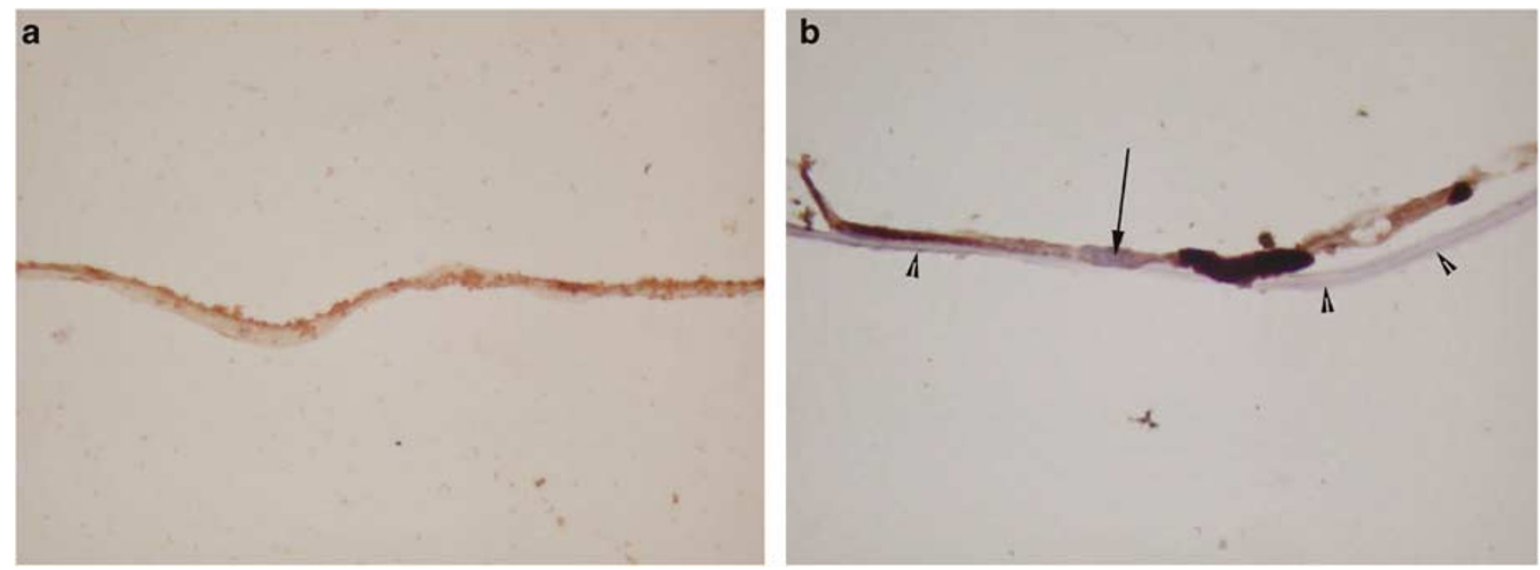

Figure 3 Immunocytochemistry of epiretinal membrane (a) (Type IV collagen) weak positive staining for type IV collagen (b) (GFAP) positive staining for GFAP in the cytoplasm of spindle cell (nucleus marked by arrow) while non cellular component of membrane was negative for GFAP (arrow heads). Magnifications a, $b \times 600$

as an amorphous smudge along one (presumably vitreous or 'nonretinal') aspect of the membrane without appearing to stain the main body of the membrane itself (not shown). The GFAP stain showed weak focal positivity marking the cells along the edge of the membrane (Figure 3b). The stain for pancytokeratin marker MNF116 was negative.

In control eyes with PVD, the posterior aspect of the detached vitreous was clearly visible as a convoluted membrane, the PHM. This membrane contained occasional cells with spindle or oval-shaped nuclei that were strikingly similar in appearance to the cell population in the above specimens (Figure 4). In some cases, the PHM was particularly thick and cellular, resembling detached retinal inner limiting membrane (ILM) (Figure 4a). With immunocytochemistry, the PHM demonstrated focal positivity for GFAP (marking the spindle cells) and type IV collagen, (Figure $4 \mathrm{~b}$ and c), but positivity for these stains was variable, as we have previously reported. ${ }^{7}$ The convoluted nature of the PHM, coupled with the two-dimensional view seen in microtomy sections made it impossible to assess which segment of the PHM overlay the macular before detachment occurred. The cells in the PHM were more easily seen in the membranes removed from the vitreous chamber following trephining of the posterior sclera. In these specimens, the Weiss ring was often visible. The cells in the membrane were most densely populated around this ring, and became increasingly sparse further from the posterior pole. In addition, pigment-laden cells and occasional inflammatory cells could be seen around the Weiss ring in some cases. These additional cell components were absent away from the ring margin.

\section{Discussion}

The pathogenesis of $\mathrm{MH}$ is yet to be completely understood and is subject to debate. Gass proposed a 

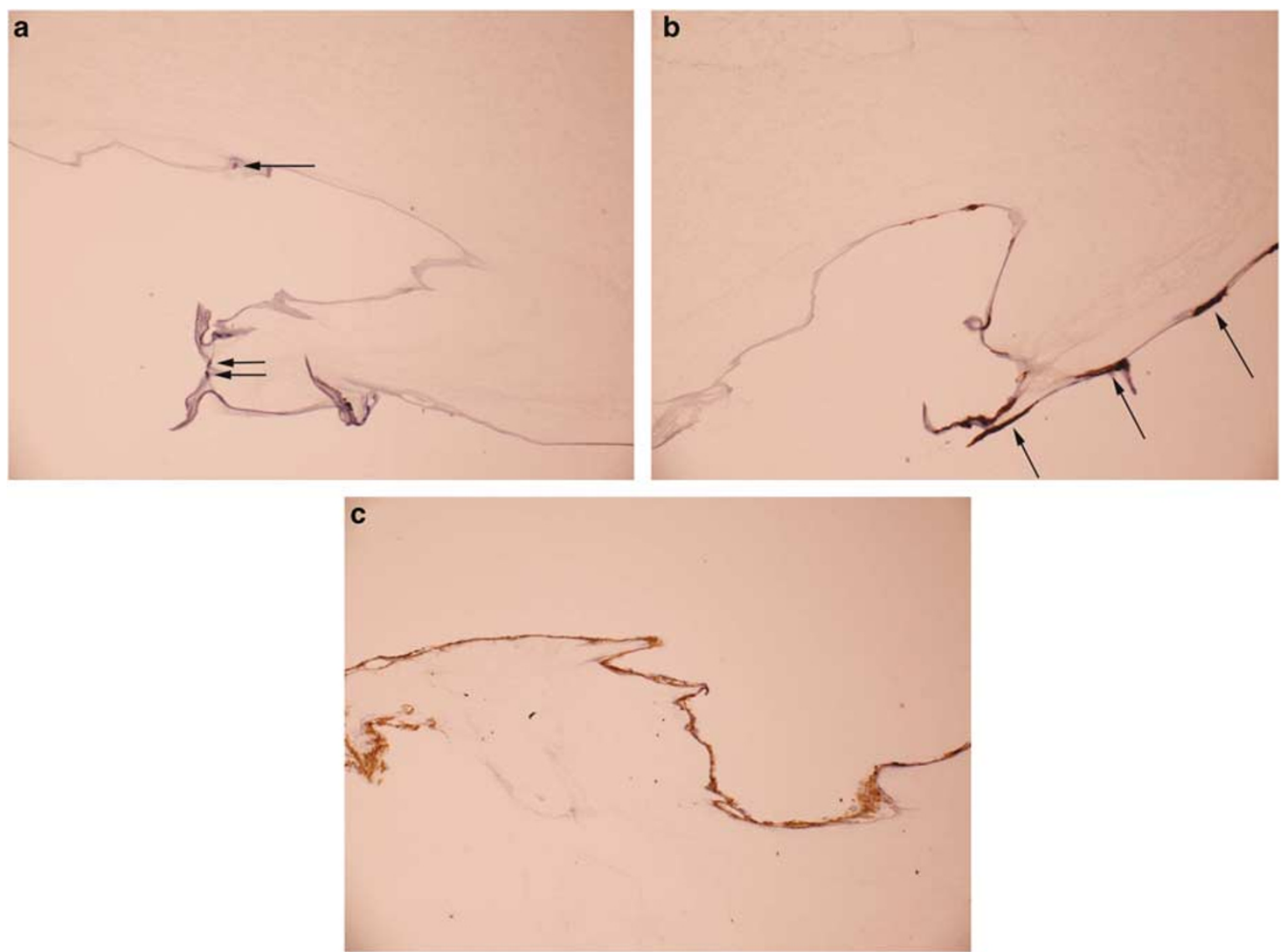

Figure 4 Histology of posterior hyaloid membrane (PHM) from cadaver control: (a) (H\&E) detached PHM covering the posterior surface of the residual vitreous cortex with cell nuclei clearly visible (arrows) along the membrane. (b) (GFAP) strong positive staining for GFAP is seen in these cells (arrows). (c) (Type IV Collagen) the PHM shows strong positive staining for type IV collagen. Magnifications a, b, c $\times 200$.

classification of idiopathic $\mathrm{MH}$ that has been widely adopted. ${ }^{8}$ Stage $1 \mathrm{~A}$ and $1 \mathrm{~B}$ lesions were thought to represent focal foveal detachments with the development of a full thickness dehiscence heralding stage 2 . In both stages, the vitreous gel was attached, and it was postulated that tangentially orientated contraction of the prefoveal cortical vitreous might be responsible for the clinical changes. In stage 3 , the vitreous gel remains attached, but with some cases showing vitreofoveal separation possibly implying the earliest stages of PVD. The development of PVD signified transition to stage 4 .

The surgical rationale for 'early' (stages 1-3) MHs has now been established. Extrapolating Gass' theories of pathogenesis into surgical practice, Kelly and co-workers ${ }^{2,3}$ pioneered successful $\mathrm{MH}$ closure using vitrectomy to induce and complete the process of PVD, and gas tamponade to maintain hole closure in the immediate postoperative period. Providing the initiating tangential shortening has been successfully eliminated, $\mathrm{MH}$ closure is maintained even after the effect of the internal tamponade has expired. Many single- and multicentre trials have followed, which generally suggest that early surgery ( $<6$ months symptoms, small holes) and adequate postoperative tamponade of the $\mathrm{MH}$ are important factors associated with successful closure. ${ }^{9-13}$

Late, chronic stage 4 holes have the least successful surgical outcome. In many series, the individual proportion of stage 4 holes is not clearly specified. However, where these data are reported, successful closure appears to be more difficult to achieve. ${ }^{14}$ Furthermore, the rationale of surgery to separate and remove vitreous from the retinal surface in these cases sits rather uneasily alongside Gass' theorem, since the vitreous has (by common consenus) already separated from the retina.

It is commonly accepted that the presence of a Weiss' ring is evidence of PVD. However, we feel that the identification of a defect-free PHM is a far more accurate indication of complete vitreous separation and this is of particular relevance in patients with stage $4 \mathrm{MH}$. For 
instance, the Weiss' ring may be destroyed during the process of posterior vitreous separation. Alternatively, even though the PHM may separate cleanly from the optic disc resulting in the formation of a Weiss ring, a further dehiscence in the neighbouring PHM overlying the macula can still result in an incomplete separation from the posterior pole. In this study, a defect could be demonstrated in the PHM in all recruited patients. A possible reason for the dehiscence of the PHM over the macula may be the presence of attachment plaques between the vitreous and retinal surface in that area only. $^{15}$

The presence of ERM could be identified in all our patients preoperatively. Histologically, the appearances of the ERM peeled were comparable to specimens removed in other studies. ${ }^{16,17}$ The ERM specimens also showed resemblance in their histological and immunocytochemical characteristics with PHM specimens obtained in a previous study. ${ }^{7,18}$ They both contain type IV collagen and GFAP-positive cells. This supports our clinical impression that during the process of PVD in eyes with stage IV MH, dehiscence of the PHM over the macula results in remnants being left and manifesting as ERM. Further support for this hypothesis is provided by an electron microscopy study by Kishi et $a l^{19}$ who found vitreous remnants overlying the fovea in $44 \%$ of autopsy eyes with PVD.

The expression of GFAP in the cells associated with the membrane suggests they are of astrocytic lineage and they have been commonly referred to as simple glial ERMs ${ }^{20}$ However, their frequent occurrence at the vitreoretinal interface has led us to suggest that such cells (also called laminocytes) may be a normal component of the inner limiting membrane of the retina. ${ }^{18}$ The presence of ILM components, that is, type IV collagen, and laminocytes, in the PHM of control PVD eyes confirms that, at least in some cases, vitreous detachment must occur beneath the level of the retinal ILM. Whether this is the case in all instances is less certain, as negative staining for ILM components could be due to loss of these antigens in eyes from patients who will usually have had their PVD many years before dying, and does not necessarily indicate separation has occurred above the ILM.

The role of ERM in the pathogenesis of $\mathrm{MH}$ remains to be resolved. The ERM specimens in a series by Messemer et al $^{16}$ were found to be highly convoluted, suggesting contracture. Similar contracture has been found in ERMs of macular pucker and cellophane maculopathy. ${ }^{21}$ This supports our clinical impression that ERM plays an important role in the maintenance of stage $4 \mathrm{MH}$. The high success rate of $\mathrm{MH}$ closure in this series also provides additional evidence of the importance of addressing the tangential force involved in $\mathrm{MH}$ formation. This is in keeping with a review of recent literature, which reveals several studies in which ERM/ ILM peeling in macular surgery has resulted in high $\mathrm{MH}$ closure rates. $^{22,23}$

In conclusion, the results of this study support our hypothesis that stage $4 \mathrm{MH}$ is associated clinically and histologically with an incomplete separation of the PHM from the retina. Surgical removal of the PHM remnant from the macula is important in the successful closure of stage $4 \mathrm{MH}$ as it plays an important role in the pathogenesis of the $\mathrm{MH}$.

\section{References}

1 Gass JD. Idiopathic senile macular hole. Its early stages and pathogenesis. Arch Ophthalmol 1988; 106: 629-639.

2 Kelly NE, Wendel RT. Vitreous surgery for idiopathic macular holes. Results of a pilot study. Arch Ophthalmol 1991; 109: 654-659.

3 Wendel RT, Patel AC, Kelly NE, Salzano TC, Wells JW, Novack GD. Vitreous surgery for macular holes. Ophthalmology 1993; 100: 1671-1676.

4 Tanner V, Chauhan DS, Jackson TL, Williamson TH. Optical coherence tomography of the vitreoretinal interface in macular hole formation. Br J Ophthalmol 2001; 85: 1092-1097.

5 Uemoto R, Yamamoto S, Aoki T, Tsukahara I, Yamamoto T, Takeuchi S. Macular configuration determined by optical coherence tomography after idiopathic macular hole surgery with or without internal limiting membrane peeling. Br J Ophthalmol 2002; 86: 1240-1242.

6 Ebato K, Kishi S. Spontaneous closure of macular hole after posterior vitreous detachment. Ophthalmic Surg Lasers 2000; 31: 245-247.

7 Snead MP, Snead DR, Mahmood AS, Scott JD. Vitreous detachment and the posterior hyaloid membrane: a clinicopathological study. Eye 1994; 8: 204-209.

8 Gass JD. Reappraisal of biomicroscopic classification of stages of development of a macular hole. Am J Ophthalmol 1995; 119: 752-759.

9 Ip MS, Baker BJ, Duker JS, Reichel E, Baumal CR, Gangnon $\mathrm{R}$ et al. Anatomical outcomes of surgery for idiopathic macular hole as determined by optical coherence tomography. Arch Ophthalmol 2002; 120: 29-35.

10 Kang HK, Chang AA, Beaumont PE. The macular hole: report of an Australian surgical series and meta-analysis of the literature. Clin Exp Ophthalmol 2000; 28: 298-308.

11 Ryan Jr EH, Gilbert HD. Results of surgical treatment of recent-onset full-thickness idiopathic macular holes. Arch Ophthalmol 1994; 112: 1545-1553.

12 Thompson JT, Smiddy WE, Glaser BM, Sjaarda RN, Flynn Jr HW. Intraocular tamponade duration and success of macular hole surgery. Retina 1996; 16: 373-382.

13 Ullrich S, Haritoglou C, Gass C, Schaumberger M, Ulbig MW, Kampik A. Macular hole size as a prognostic factor in macular hole surgery. Br J Ophthalmol 2002; 86: 390-393.

14 Gregor ZJ. Surgery for idiopathic full-thickness macular holes. Eye 1996; 10: 685-690.

15 Foos RY. Vitreoretinal juncture; topographical variations. Invest Ophthalmol 1972; 11: 801-808.

16 Messmer EM, Heidenkummer HP, Kampik A. Ultrastructure of epiretinal membranes associated with 
macular holes. Graefes Arch Clin Exp Ophthalmol 1998; 236: 248-254.

17 Li K, Wong D, Hiscott P, Stanga P, Groenewald C, McGalliard J. Trypan blue staining of internal limiting membrane and epiretinal membrane during vitrectomy: visual results and histopathological findings. $\mathrm{Br} \mathrm{J}$ Ophthalmol 2003; 87: 216-219.

18 Snead MP, Snead DR, Richards AJ, Harrison JB, Poulson AV, Morris AH et al. Clinical, histological and ultrastructural studies of the posterior hyaloid membrane. Eye 2002; 16: 447-453.

19 Kishi S, Demaria C, Shimizu K. Vitreous cortex remnants at the fovea after spontaneous vitreous detachment. Int Ophthalmol 1986; 9: 253-260.
20 Foos RY. Vitreoretinal juncture - simple epiretinal membranes. Albrecht Von Graefes Arch Klin Exp Ophthalmol 1974; 189: 231-250.

21 Snead DR, Cullen N, Poulson AV, Morris AH, Lukaris A, Scott JD et al. Hyperconvolution of the inner limiting membrane in vitreomaculopathies. Graefes Arch Clin Exp Ophthalmol 2004; 242(10): 853-862.

22 Eckardt C, Eckardt U, Groos S, Luciano L, Reale E. [Removal of the internal limiting membrane in macular holes. Clinical and morphological findings]. Ophthalmologe 1997; 94: 545-551.

23 Mester V, Kuhn F. Internal limiting membrane removal in the management of full-thickness macular holes. Am J Ophthalmol 2000; 129: 769-777. 